\title{
Les murs, le guet et la communauté : la construction d'un système défensif
}

Guilhem FERRAND ${ }^{1}$

\section{Résumé.}

La mise en défense des agglomérations à la fin du Moyen Âge a souvent consisté à ériger une enceinte autour de celles-ci. L'étude de cette dernière oblige à mêler sources écrites et sources archéologiques, sans que la confrontation soit toujours probante. Au final, s'il est possible de se faire une idée de la structure matérielle des fortifications, une question surtout se pose : qu'est-ce qu'est véritablement l'enceinte ? Elle n'est qu'un élément d'un système défensif plus global. Celuici associe aux murs un service de guet, et relie la protection de la ville à celle de son territoire. L'information apparaît dans ce système comme l'élément central de la défense. Ce système est un système urbain. Dans le "plat pays, » la défense a pu revêtir d'autres formes, sans qu'il soit toujours possible de les mettre au jour efficacement. Cohabitent ainsi plusieurs systèmes que l'historien aujourd'hui a souvent du mal à relier entre eux, pour des raisons documentaires essentiellement. Ces différentes formes obligent toutefois à poser une question centrale : la mise en défense va-t-elle de soi ?

Mots-clés :Guerre de Cent Ans, enceinte, guet, système défensif, monde urbain, "plat pays. »

Le 27 juin 1418, le comte d'Armagnac écrit aux consuls du Bourg de Rodez, dont il est le seigneur, qu'il va bien". "Nous avons pris et brûlé le lieu de Servayreta, ajoute-t-il ; les ennemis sont défaits et la plupart sont morts, ou pris ; quelques-uns ont fui à Montauros et à Sant-Daunis.» C'est pourquoi, avec toute sa suite d'hommes d'armes, il part assiéger les lieux en question ${ }^{3}$. L'épisode résume assez bien certaines des réalités militaires courantes pendant la guerre de Cent Ans : des agglomérations fortifiées, où s'abritent soldats et population, que des troupes ennemies assiègent.

La mise en défense des villes est un phénomène historique mis au jour depuis longtemps. De nombreux travaux l'attestent, monographies urbaines ou régionales. Plusieurs synthèses récentes en rendent compte, qui dessinent assez bien un schéma d'ensemble (Blieck, Contamine, Faucherre et Mesqui 1999; Butaud 2002; Desplat 2002; Le Blévec 2002). Bon nombre d'agglomérations s'emmurent au milieu du $\mathrm{XIV}^{\mathrm{e}}$ siècle. Elles disposent parfois des restes d'une enceinte, érigée en d'autres temps, mais souvent mal entretenue depuis. De gros travaux sont engagés pour mettre la ville en défense. La première difficulté - la seule, sans doute - est de trouver les moyens financiers requis (Contamine 1978; Rigaudière 1993 [1984]). La mise en défense matérielle s'accompagne d'autres mesures, dont l'activation ou la ré-activation du guet est la principale. La ville dispose alors à la fois de l'information et des infrastructures nécessaires à sa défense: sa sécurité est assurée. L'entretien des murs pose toutefois problème: les travaux succèdent aux travaux, d'une manière presque incessante. L'adaptation des infrastructures aux

\footnotetext{
${ }^{1}$ Allocataire-moniteur, Université de Toulouse, CNRS - Framespa/Terrae, guilhem.ferrand@wanadoo.fr.

2 Rodez est une ville double : le comte est seigneur du Bourg, l'évêque est seigneur de la Cité.

${ }^{3}$ A.D. Aveyron 2 E 212 Bourg CC 131, fo $31 \mathrm{r}^{\circ}$ : «Item a XXV II de jun venc un escudier apelat Alfanso, loqual portet novelas e letras de mossenhor Bernat d'Armanhac a Madama, et letras als cossols de Borc e de Cieutat, per las quals mandava lo bon estat de sa persona, et cum avian pres lo loc de Servayreta e cremat e los enemix escofitz et la plus grant partida mortz o pres, et l'autra partida s'en eron fugitz a Montauros et a Sant Daunis, els quals locz lo dich mossenhor Bernat am sa tres nobla companbia los anavan assalir et assetiar; fon donat al dich escudier entre nos et los de Cieutat II l. ; paguem per nostra part una lieura. »
} 
évolutions de l'armement fait aussi difficulté, ce qui explique parfois la réfection complète de l'enceinte.

La mise en défense d'une agglomération, quelle que soit sa taille, est l'une des formes, et l'une d'elles seulement, que prend la défense des communautés. C'est cette forme-là que je voudrais ici, à partir de trois dossiers rouergats - Espalion, Rodez et Villefranche-de-Rouergue -, regarder en détail. Plusieurs problèmes sautent aux yeux, qui relèvent tous, me semble-t-il, d'une seule et même question que l'on peut qualifier, de prime abord, de documentaire: quelles archives subsiste-t-il pour quelle réalité historique? Il y a - c'est évident - les sources écrites et les archives du sol, lacunaires dans les deux cas. Ces lacunes, je crois, n'ont pas suffisamment été prises en compte: le cas de la mise en défense des agglomérations le montre assez bien. Ce faisant, il révèle d'une manière éclatante un problème d'ordre épistémologique ou méthodologique, sournois mais fondamental : quelle que soit la forme que la mise en défense des communautés et des hommes a pu prendre, son approche est tributaire des sources disponibles aujourd'hui. La contrainte que celles-ci font peser sur l'appréhension du phénomène est le premier élément à verser au dossier de son étude.

\section{L'enceinte.}

D'où partir, sinon de l'enceinte elle-même? La bibliographie disponible permet aujourd'hui de se faire une idée parfois précise de sa réalité matérielle, de sa mise en œuvre, des grandes lignes de son évolution, quand des réfections d'importance ont modifié à dessein le programme initial. Jean Mesqui, en 1979, déplorait le manque d'études strictement archéologiques, au profit, selon lui, d'études historiques prenant mal en compte l'aspect matériel de l'enceinte (Mesqui 1979, p. 2). Le fossé, aujourd'hui encore, est mal comblé, ce qu'écrit Jean Mesqui lui-même dans l'introduction du colloque du C.T.H.S. de 1996 consacré aux enceintes urbaines: l'absence de vestiges en élévation comme la difficulté d'opérer des fouilles en milieu urbain interdisent bien souvent d'approcher de près la fortification (Blieck, Contamine, Faucherre et Mesqui 1999, p. 8). Conserver des vestiges ne suffit pas : encore faut-il pouvoir en tirer des informations. Sans disposer toujours d'une stratigraphie parlante, face au mur nu, muet lui aussi, que peut dire l'archéologue? Le cas de l'étude de l'enceinte villageoise de Peyrelade, dans l'Aveyron, illustre bien le problème: "l'enceinte primitive de Peyrelade, c'est-à-dire celle du château, fut très certainement construite vers le $\mathrm{XII}^{\mathrm{e}}$ siècle. Celle beaucoup plus conséquente qui enferme le village est sans nul doute à relier, d'une manière historique, à la guerre de Cent Ans qui vit de nombreux sites aveyronnais se fortifier. Elle peut donc être datée du XIV e siècle » (Poujol et Simonin 1997, p. 21). Le propos parait caricatural. Je ne suis pas sûr, pourtant, qu'il le soit vraiment. De quoi dispose Christophe Sireix à Rodez, par exemple, pour regarder de près le rempart médiéval retrouvé à l'occasion de sondages effectués sur le site du Parmentier, boulevard Laromiguière (Sireix 1997) ? D'un mur, isolé de l'ensemble auquel il appartenait, bien conservé à l'évidence, mais que la structure ne permet pas de dater; elle ne permet pas non plus d'y lire d'éventuelles reprises, à quelques détails près qui ne paraissent pas utilisables (Fig. 1). D'une stratigraphie limitée, côté nord-est du mur seulement, mais suffisante, à partir d'indices bien ténus cependant, pour dater le mur du $\mathrm{XIV}^{\mathrm{e}}$ siècle et distinguer deux phases de construction assez nettes : une tranchée étroite (U.S. 2003 à 2005) creusée au moment de la fondation du mur ; une tranchée large (U.S. 2001), remplie au fur et à mesure de la construction, témoin d'un deuxième moment de l'entreprise (Fig. 2). C'est mince. L'étude de l'enceinte n'a de sens que lorsqu'il est possible de croiser différentes sources d'information : comptabilité urbaine souvent, prix-faits et vestiges, à supposer que ces derniers puissent sans risque être reliés aux registres et documents à la disposition du chercheur. 


\section{Espalion.}

L'exemple d'Espalion, petite ville dans le nord de l'Aveyron actuel, au bord du Lot, me parait être un exemple relativement représentatif de ce que l'on peut savoir d'ordinaire, dans la majeure partie des cas.

La documentation écrite, sans être abondante, est tout de même fournie : peu de choses pour le $\mathrm{XIV}^{\mathrm{e}}$ siècle et les périodes antérieures, mais des indices probants de l'existence d'une enceinte ou de quelque chose d'approchant dès le milieu du XIII ${ }^{\mathrm{e}}$ siècle au moins : il est question de clés et de portes dans la charte de coutumes de 1266. Un document de 1367 - octroi du souquet ou gabelle par le seigneur de la ville, le seigneur de Calmont d'Olt - renseigne plus ou moins sur le moment de la mise en œuvre ou de sa révision globale : les consuls ont besoin d'argent pour réparer l'existant et compléter le dispositif défensif. La documentation du $\mathrm{XV}^{\mathrm{e}}$ siècle permet de se faire une idée précise de son emprise. Quatre registres d'estimes conservés $(1403,1435,1461$ et les années 1490) offrent quatre clichés de la ville, certes un peu flous, mais précieux. Ils permettent de dessiner les contours de l'enceinte et, pour partie au moins, de son évolution. Plusieurs vestiges conservés en élévation, pans de murs (Fig. $3 \mathrm{a}$ et b) et tours d'angle (Fig. 4), confirment le tracé et donnent une idée de l'aspect matériel (Ferrand 2001).

Au total, que savons-nous? L'enceinte, revue et complétée sans doute au milieu du XIV siècle, enserre la ville (Fig. $5 \mathrm{a}$ et b). Elle comprend quatre tours d'angle. La porte principale, au sud-ouest, est protégée par une barbacane, une autre porte, au sud-est, par une passerelle (planca) aisément amovible dans l'urgence. La troisième porte est l'ensemble formé par le pont, bâti et fortifié. Une partie des faubourgs est fortifiée, probablement au milieu du $\mathrm{XV}^{\mathrm{e}}$ siècle. Un tableau de 1653 délivre un témoignage iconographique de premier ordre, même s'il rend compte aussi, dans le même temps, des restructurations postérieures : destruction de l'une des tours d'angle au profit d'un palais, celui du gouverneur militaire de la ville dans la deuxième moitié du XVI siècle ; construction probable d'un bastion, à proximité de l'une des autres tours d'angle... (Fig. 6). Les comptes consulaires, conservés très partiellement pour la deuxième moitié du $\mathrm{XV}^{\mathrm{e}}$ siècle, font état d'un vaste glacis protecteur autour de la ville englobant à la fois les fossés et, au sud-est, la bande de terre comprise entre le mur et le fossé. Plusieurs mentions rendent compte des difficultés financières importantes auxquelles la communauté est confrontée, qui gênent l'entretien de l'enceinte.

La vue d'ensemble est incomplète, mais la documentation est suffisante, je crois, pour se faire une idée un peu précise de la structure matérielle qui protège la ville.

\section{La Cité de Rodez.}

Une documentation plus fournie, si elle permet d'aller plus loin, de définir plus précisément le programme initial mis en œuvre et les réfections d'ensemble qui ont pu suivre, permet surtout de mettre au jour un problème d'importance. L'exemple de la Cité de Rodez, en Rouergue, peut servir de révélateur.

La comptabilité urbaine des consuls de la Cité, conservée depuis 1350, est presque continue entre 1380 et 1450 . Seuls manquent à l'appel, pour cette période-là, huit exercices. Chacun de ces bilans comptables, rédigés par le trésorier de la ville, est chapitré. L'un de ces chapitres concerne les dépenses effectuées pour les bâtiments : "Ensec si la despensa facha per las obras de la viela.» Au gré de la lecture des items, on peut se faire une idée précise de l'entretien, presque au quotidien, qu'exige l'enceinte.

L'exemple de l'entretien d'une porte, point crucial de l'enceinte, celle de Penavayre, située au sud de la cathédrale, me parait éloquent (Fig. 7). Sur vingt ans, du réarmement massif de $1408^{4}$

\footnotetext{
${ }^{4}$ Après une longue pause, plusieurs événements, dont l'assasinat du duc d'Orléans en novembre 1407, mettent à nouveau le feu aux poudres. À Rodez - les comptes consulaires de la Cité en rendent compte -, le «basculement »
} 
à la fin des années 1420 , on voit que des réparations sont très régulièrement effectuées. Souvent, il ne s'agit que de menues réparations. On peut objecter, à l'instar de Germain Butaud, que changer chaque année la serrure de la porte n'est pas d'une très grande importance et ne modifie pas grand chose, ni la porte, ni son fonctionnement (Butaud 2002, p. 241). Regarder de près certaines de ces réparations - les plus sérieuses - pour l'ensemble de l'enceinte sur une décennie, celle des années 1430 par exemple, à un moment où règne une très grande insécurité générée en particulier par Rodrigue de Villandrando et sa compagnie, oblige à changer de point de vue (Fig. 8). La lecture du tableau suffit à poser le problème: qu'est-ce qu'une enceinte dès lors que régulièrement, très souvent même, une brèche se forme, un pan de mur s'affaisse, une tour s'effondre, que tel ou tel passage que doit emprunter le guet est impraticable? Que sont les portes où les serrures sont à changer constamment ? Bref, qu'est-ce qu'une enceinte? Jean-Pierre Leguay, récemment, a posé le problème: «La chute d'une courtine n'a pas que des conséquences matérielles et financières. Elle renforce le climat d'insécurité, traumatise une population profondément attachée à des défenses payées de ses deniers, brise l'image d'invulnérabilité que les anecdotes locales ont tendance à accréditer et à transmettre à la postérité » (Leguay 2005, p. 127 et 128). Gilles Blieck, travaillant sur Lille, estime que l'entretien courant n'est pas utile à regarder pour qui travaille sur l'enceinte. Il préfère les grands moments où les plans s'élaborent et se mettent en œuvre, qui délivrent une vue d'ensemble du dispositif: "Menés au gré des circonstances et surtout des possibilités financières, les travaux de strict entretien ne sont pas, en raison de leur caractère ponctuel, les plus propices à une analyse très poussée. L'on se met très vite à rechercher les moments privilégiés, où une brusque menace conduit à renforcer l'ensemble des défenses et à les mettre au goût du jour, offrant du même coup l'opportunité d'une vision globale à un moment donné » (Blieck 1998, p. 195). Bien à tort : les détails sont ponctuels, certes, et ne permettent pas d'appréhender l'enceinte dans son ensemble, mais ils révèlent un problème au cœur de l'investigation en mettant en question la définition même de l'objet d'étude. Y a-t-il lieu, d'ailleurs, sur ce point précis, d'opposer détails et grands moments? Tous les auteurs s'accordent à parler de chantiers longs : sur plus d'une génération à Orléans (Michaud-Fréjaville 1981), sur plus de trente ans aussi au Quesnoy (Salamagne 1981), sur plus de dix ans au moins à Avignon (Hayez 1978)... etc. Décidée dans l'urgence, la construction de l'enceinte, ou sa révision complète, s'opère sur plusieurs années - le problème est le même qu'il s'agisse de quelques mois ou de plusieurs années -. Comment assure-t-on la défense de la communauté, dans l'intervalle? Germain Butaud explique que "pendant l'édification de la nouvelle enceinte, la défense de la ville était assurée, en plus du fossé, par un ensemble de fortifications provisoires qui étaient levées entre les portes ou les pans de remparts définitifs en pierre ou en brique.» Pour lui, «ces défenses, nécessaires pour clore la ville dans sa totalité et parer aux menaces immédiates, étaient ensuite détruites au fur et à mesure que le chantier progressait» (Butaud 2002, p. 238). Philippe Lardin évoque des défenses de ce type à Dieppe : leur existence, le mur principal achevé ou non, permettait de considérer la ville comme une "ville fermée " (Lardin 2006, p. 98). À Bordeaux, pendant la guerre de Saint-Sardos, en 1324, alors que la ville vit dans la crainte d'une attaque imminente, il est décidé de construire une batterie de machines de guerre pour suppléer, côté Garonne, à l'absence de mur (Bériac et Hanusse 1992). Le problème me paraît donc être identique: dès lors qu'une structure provisoire peut suffire à stopper l'ennemi, qu'est-ce que l'enceinte? S'interroger de la sorte rejoint le désir exprimé avec force par Philippe Contamine: celui d'appréhender l'enceinte non pas en elle-même, d'un point de vue structurel, mais en action (Contamine 1999, p. 331 ; Contamine 2002b, p. 198). Les dispositions qui accompagnent l'érection ou la réfection du mur préparent l'action. Elles en disent assez long sur le mur luimême. La principale de ces dispositions est la mise en place d'un guet.

dans une nouvelle logique de guerre est très bien compris par les autorités consulaires. Celles-ci procèdent, dans les premiers mois de l'année 1408, à un réarmement massif de la ville. Des travaux sont engagés, sur trois ou quatre ans. 


\section{Le guet.}

En temps de guerre, que la menace soit réelle ou anticipée avec sérieux, sur la muraille érigée à grand peine et à grands frais, la communauté ou l'autorité placent des sentinelles. La muraille seule ne sert à rien. Sa construction ou sa réfection va de pair avec l'activation ou la réactivation d'un service de guet. Il n'est pas toujours possible d'approcher avec précision les modalités de l'organisation de celui-ci : quelques mentions éparses confirment son existence sans expliquer tout à fait la manière dont il fonctionne. Un texte d'ordre normatif, conservé pour Villefranche-de-Rouergue, éclaire le phénomène.

\section{Le guet, la garde et les portes : l'exemple de Villefranche-de-Rouergue.}

Le texte est une copie effectuée au XVII ${ }^{\mathrm{e}}$ siècle par l'équipe du président Doat d'un document conservé à l'hôtel de ville de Villefranche-de-Rouergue (A.N., Fonds Doat, reg. 147, fo $282 \mathrm{r}^{\circ}$ à $287 \mathrm{r}^{\circ}$; annexe, p. XXX à XXX). Il n'est pas daté. Aucune précision ne permet de savoir de quoi il s'agit. Le style est celui d'une ordonnance consulaire. Plusieurs expressions laissent penser qu'il s'agit d'un texte importé d'ailleurs et repris à leur compte par les consuls de la ville. Au fond, peu importe : il décrit le fonctionnement de la défense d'un point de vue théorique, tel que les consuls de Villefranche-de-Rouergue l'ont pensé ou tel qu'ils s'en sont appropriés la conception.

Les instructions que le texte donne sont conformes à celles qu'Aristote envoya à Alexandre pour défendre Bucifala. Cette allusion à l'Antiquité suffit à résumer la philosophie d'ensemble du document. Dans le Roman d'Alexandre, en effet - sans remonter plus haut dans la littérature -, la ville de Bucifala est présentée commme la ville-forteresse imprenable, parfaite au moins d'un point de vue défensif :

«Quant ele fu fondee, tours i ot cent et set,

Ne crient assaut ne siege ne tornoi ne aguet,

Perriere ne berfroi n'engin qui ja soit fet.

Molt fu la cité bele et sist en un desert»

(Alexandre de Paris 1994, \245, vers 4420 à 4423, p. 572). Assurer totalement la défense de la ville est bien ce à quoi prétend le document, agençant tout un système défensif. Tout le monde, dans la ville menacée, est concerné : chacun joue un rôle. La ville, prévoyante, a construit des murailles qu'elle a dotées de structures défensives : tours de guet et créneaux. Elle a protégé les abords des murs par un fossé et des ouvrages défensifs à proximité. Elle a mis en place un service de guet qui assure une présence constante sur l'enceinte où des chaudrons pleins d'eau prête à bouillir, de la chaux et des pierres à lancer attendent l'action. Il y a donc des hommes sur les murs, qui ne peuvent laisser leurs postes, "a pena de la testa.» Ils sont soutenus par des hommes d'armes à cheval prêts à effectuer une sortie en dehors de la ville. Des hommes patrouillent dans les rues pour veiller à la mobilisation de chacun, et apporter réconfort, vivres et munitions là où nécessaire, secondés sur ce point par les femmes - "totas las femmes.» En cas de besoin, des éléments défensifs peuvent être construits bien au-delà des murs. L'ennemi s'annonçant, des messagers sont envoyés vers lui, chargés de mener de fausses négociations, le temps pour les habitants de la ville de finir de s'organiser, de mettre l'eau et la chaux à chauffer et d'apprêter les crochets et les marteaux de fer avec lesquels repousser les échelles de l'assaillant, le temps surtout pour les messagers de se renseigner sur les troupes ennemies, "quan de gens so ni que volo far. »

Ce dont parle ce texte, c'est d'un système défensif, qui ne limite pas la défense de la ville à à sa seule muraille, mais qui relie l'agglomération à ses alentours et qui accorde à l'information une place de choix. 


\section{Un système défensif.}

La plupart des travaux consacrés à la mise en défense de la ville ont montré, d'une manière plus ou moins détaillée, comment s'agençait le système défensif, même si la documentation conservée ne permet pas toujours de bien en mesurer l'ampleur. L'une des énonciations les plus significatives me parait être ce que Michel Hébert montre pour Tarascon à la fin du XIV es siècle (Hébert 1978, p. 170 à 175). Il distingue plusieurs volets. D'une part la cité se replie sur elle-même, derrière ses murs. D'autre part, elle met en place une garnison et arme l'ensemble de la population. Elle cherche par ailleurs à préserver la cohérence de la communauté, renforçant l'exclusion des marginaux. De la sorte, «la ville dispose d'un système complet d'autodéfense : rupture des contacts avec l'extérieur, mobilisation et encadrement de la population, renforcement des liens de solidarité interne. » Pour autant, ce n'est pas suffisant. La ville n'est pas simplement la zone délimitée par les remparts : elle s'inscrit dans un territoire. "Cette proche banlieue est essentielle à la survie des habitants, mais sa mise en état de défense suppose une organisation beaucoup plus complexe pour laquelle la plupart du temps la population tarasconnaise doit faire appel à des gens d'armes de l'extérieur. » La ville cherche à protéger ce territoire en créant quelques points d'appui fortifiés, où elle place une garnison. Elle détruit ce qui est inutile et qui lui paraît dangereux, à même de servir à l'ennemi. Elle met en place, enfin, un réseau de surveillance et d'information à l'échelle de la viguerie, et demande au sénéchal, pour subvenir aux besoins que cette surveillance génère, l'autorisation de prélever une taxe spéciale sur l'ensemble des habitants de la viguerie, puisque, dans les faits, il lui semble que la viguerie tout entière tire profit du système. Bernard Fournioux a montré, pour la châtellenie de Montignac, dans le Périgord, au XIII ${ }^{\mathrm{e}}$ siècle surtout mais aussi à la fin du Moyen Âge, que l'agglomération principale est bien au centre du réseau de surveillance, mais que le réseau sert à tous, diffusant l'information en moins d'un quart d'heure d'un bout à l'autre de la châtellenie (Fournioux 1990).

Un système défensif du même type, ou approchant, a été récemment mis en évidence pour Cahors par Nicolas Savy, accordant une large place à une dimension supplémentaire du système : la ville de Cahors inscrit son action dans un territoire plus vaste, au sein duquel s'opère une collaboration entre les différentes agglomérations (Savy 2005). Pour Pierre Flandin-Bléty, c'est d'ailleurs ce travail collectif étroit, lié à la défense du pays, qui a généré l'idée d'un corps à laquelle chacune des villes estimait appartenir. Il voit là, pour le Quercy et le Rouergue, la naissance du Tiers État (Flandin-Bléty 1979).

À bien y regarder, c'est donc bien d'un système défensif qu'il s'agit, dans lequel le territoire, la surveillance et la maitrise de l'information paraissent, tout autant que l'enceinte, les éléments structurants. Toutefois, de quel système défensif parle-t-on vraiment? Ce système défensif est urbain et ne concerne a priori que les villes, petites ou grandes.

\section{La ville et le plat pays.}

Le système défensif, tel qu'on le connaît, tel qu'il est possible de le mettre au jour sans trop de difficulté est un schéma urbain, ce que résume très bien l'expression d' "enceinte urbaine.» Il met en œuvre les agglomérations, au sens large du terme, considérées à la fois individuellement et en réseau, et leurs territoires respectifs. Le reste, c'est le "plat pays.» L'opposition me pose problème, comme l'opposition presque systématique, quelle que soit la question en histoire médiévale, entre monde urbain et monde rural. Des différences existent, qu'il est difficile de nier. La séparation, c'est-à-dire l'absence d'approche globale, oblitère cependant, à mon sens, la compréhension du phénomène à observer.

La principale différence, outre les structures et les moyens défensifs mis en œuvre, est, pour nous, chercheurs d'aujourd'hui, une fois de plus, un problème d'ordre documentaire. Plusieurs vues d'ensemble disponibles, régionales ou générales, attirent l'attention, que cela soit formalisé ou non, d'abord et avant tout sur ce point (Gaier 1995 [1963] ; Fournier 1966 ; 
Lartigaut 1967 ; Contamine 2002a ; Loppe 2004 ; Germain 2005). Que sait-on? Que peut-on savoir? Peu de choses, à vrai dire : une idée des différentes structures défensives utilisées, dont témoigne la typologie dressée par Gabriel Fournier pour la Basse-Auvergne, le travail de Claude Gaier pour la région mosane ou celui de René Germain pour le duché de Bourbon ; une idée du maillage défensif, dont rend compte l'inventaire établi à grand peine par Jean Lartigaut pour le Quercy occidental. Le travail de Jean Lartigaut est particulièrement instructif sur la question documentaire : il s'est efforcé de glaner partout où cela était possible des informations, regrettant au passage de ne pas pouvoir disposer véritablement de données archéologiques. Plus complet sans doute, tributaire d'une meilleure documentation, l'inventaire de Frédéric Loppe pour la région de Lagrasse, dans l'Aude, n'offre pas véritablement une vue d'ensemble beaucoup plus riche. La documentation fait défaut, pour le plat pays, et permet, dans le meilleur des cas, de recenser les lieux fortifiés, sans qu'il soit possible de savoir si la liste est complète. Une liste de points forts, donc, établie avec difficultés, au sein de laquelle agglomérations et châteaux occupent les premiers rangs. À coup sûr, la vision obtenue est floue. Est-ce un problème ? Il est tentant de se simplifier la besogne et d'expliquer que quelques lieux refuge parsemés sur tout le territoire suffisent à protéger l'ensemble de la population. Bernard Fournioux résume assez bien l'idée. À ses yeux, la mise en place d'un réseau d'information couvrant l'ensemble de la châtellenie de Montignac implique l'existence d'un réseau de lieux refuge "discrets, » à même de protéger l'ensemble de la population du territoire. Il pense en particulier à certains clusaux qui ont pu servir à abriter des individus (Fournioux 1990, p. 337 et 338). Marc Bloch et Philippe Contamine évoquent pour leur part l'existence d'autres structures que les agglomérations fortifiées, les châteaux, les forts et les églises : des grottes ou des cavernes, des cabanes dans les bois, des îles de certains cours d'eau (Bloch 1952 [1931], p. 118 ; Contamine 2002a, p. 29)... Ces évocations sont sans doute à peu près la seule chose qui peut subsister de ces structures, même si, ici et là, quelques mentions écrites en font état. Ces évocations suffisent à révéler les pans entiers du problème qui échappent au regard, parce que mal renseignés. À défaut, à oublier ces pratiques défensives, est-on bien sûr d'avoir compris ce qui se joue en matière de mise en défense?

\section{L'Espalionnais (Fig. 9).}

L'Espalionnais permet d'illustrer le problème et de le poser en termes un peu plus précis. La haute vallée du Lot comprend une succession de petites villes en partie fortifiées, ou du moins protégées. Je ne reviens pas sur le cas d'Espalion. En amont, la petite ville de Saint-Côme est dotée de murailles. L'ensemble du système défensif est revu en 1366, à la demande du seigneur de la ville, le seigneur de Calmont d'Olt: les fossés sont nettoyés, les murailles réparées, les points faibles renforcés, le guet restructuré... (Carnus et Cabanettes 1966, p. 40). En aval, la ville d'Estaing est en partie protégée par le château qui la domine, doté d'une vaste basse-cour. Une partie de l'agglomération seulement semble avoir été fortifiée : le quartier de la rue d'Outre (Ginisty 1975, p. 109). Trois agglomérations, donc, au bord du Lot, à même d'assurer la protection d'une population importante. Quelle population y trouve refuge ? C'est une question à laquelle je n'ai pas de réponse. Les comptes consulaires d'Espalion, partiellement conservés pour la deuxième moitié du $\mathrm{XV}^{\mathrm{e}}$ siècle, livrent quelques indications concernant un bassin sécuritaire, dont la ville s'estime en droit, en contre-partie, d'exiger fourniture de vivres et défraiements pour le logement des gens d'armes. Cela n'est pas suffisant pour dessiner avec précision les contours d'un territoire. L'absence de documentation explicite empêche de se faire une idée pour SaintCôme-d'Olt et Estaing. Ici et là, on connaît quelques lieux fortifiés : un fort à Coubisou ou à Flaujac, un château à Cabrespines, à Saint-Pierre de Bessuéjouls ou à Roquelaure, par exemple ; plus en hauteur, l'église fortifiée d'Anglars, la tour de Masse, grange fortifiée de l'abbaye de Bonneval... etc. La liste n'est pas très longue; elle est incomplète. Rien ne permet de dire, par exemple, avec certitude, comment se protégeaient ceux qui habitaient la vallée du ruisseau de Perse, en amont d'Espalion. Je doute qu'ils aient pu gagner, dans l'urgence, les lieux fortifiés 
avoisinant connus. Peut-être se réfugiaient-ils dans les bois, peut-être pas. Du coup, quoi faire? On peut se limiter à l'existant, et brosser le tableau suivant : celui de petites agglomérations bien défendues, assurant chacune la protection d'un territoire plus ou moins défini; celui de lieux fortifiés, ici et là, participant d'un maillage défensif ; celui d'espaces pour lesquels on ne sait rien. Pourtant, si la documentation conservée n'est pas complète et ne permet pas de dessiner une vue d'ensemble exhaustive, elle indique tout de même les grandes lignes du tableau. On ne sait rien sur la vallée du ruisseau de Perse, mais quels documents auraient pu, conservés, nous renseigner? Quel lieu refuge spécifique construit aurait pu ne laisser aucune trace aujourd'hui? Il est difficile d'aller plus loin. Sauf à poser la question de savoir si la mise en défense va de soi.

\section{Quelle mise en défense?}

Flaujac est un petit village à la limite du territoire d'Espalion et de celui de Saint-Côme, au bord de la boralde flaujagaise, frontière connue entre deux juridictions consulaires qui ont du mal à cohabiter. Ses habitants relèvent de la seigneurie de Calmont d'Olt. Ils lui demandent, en $1442^{5}$, l'autorisation d'ériger un fort, demande à laquelle le seigneur accède (Affre 1858 I, p. 55 et 56). Le village est situé à moins d'une demi-heure de marche des deux agglomérations voisines. Qu'est-ce qui motive la demande ? Peut-être la grande peur générée par les agissements de Rodrigue de Villandrando et de son lieutenant Jean de Salazar, dans la décennie précédente - une grande peur qui a pu donner une autre idée des distances à ceux qui devaient, dans l'urgence, les parcourir. Peut-être des moyens financiers sont-ils disponibles, qui ne l'étaient pas, et qui permettent à cette date d'envisager la construction d'un fort. Peut-être une autre raison, ou d'autres ${ }^{6}$. L'exemple est intéressant, parce qu'il est mal renseigné, à l'exception des vestiges imposants qui subsistent. Il me semble qu'il illustre très bien l'idée que la mise en défense peut ne pas aller de soi. Certaines communautés, d'ailleurs, comme celle de Labastide-L'évêque, dans la région de Villefranche-deRouergue, ne se sont pas fortifiées - une réalité qui mérite attention.

La lecture de l'enquête conduite en 1375 pour le compte du comte d'Armagnac dans le nord de l'Aveyron actuel permet de s'interroger plus précisément encore. L'enquête est diligentée à la suite d'exactions commises par des Anglais ou des individus s'en réclamant. Des enquêteurs, mandatés par le comte, parcourent une vaste zone, interrogeant les habitants des agglomérations et les habitants des mas ${ }^{7}$. Ce sont ces derniers qui m'intéressent. La plupart des dépositions font état de pillages : qui a perdu une vache, qui a perdu un sac de froment... etc. Elles ne permettent pas de traiter la question des lieux refuges. Quelques unes, toutefois, laissent entrevoir que les témoins ont vécu la scène qu'ils rapportent.. "Jobannes Ruphi, par exemple, mansi del Forn del Mas mandamenti castri de Aurella sub ressorto comitatus ruthenae, qui deposuit anno quo supra, et die vicesima mensis praedicti, testis iuratus et interrogatus super praemissis dixit quod nuper in vesperis beatae Luciae virginis, quidam nominatus lo Picart de Carlato, cum aliis novem complicibus, ad bospicium eiusdem loquentis venit, et ipsum loquentem stando in patu de uno lodice, duobus linteaminibus, et de una securi, et una mantae, et pannis de bru, cum una armercla capitis depraedaverunt, nec non sibi loquenti depastaverunt duo sestaria avenae, et tres galinas, et certas salcissas porci, quae omnia praedicta communi estimatione valent quinque florenos auri, et etiam

\footnotetext{
${ }^{5}$ Le document est perdu. Les érudits qui en font état parlent d'une autorisation accordée le 20 mars 1442 . La date donnée est-elle celle du document ? Si c'est le cas, il faut lire 1443, l'année commençant en Rouergue au 25 mars.

${ }^{6}$ Lors de la discussion qui a suivi la communication, le 20 avril 2007, Vincent Challet s'est demandé s'il ne pouvait pas s'agir d'une reconstruction plutôt que d'une construction. Il a rappelé qu'à La Couvertoirade, par exemple, un premier système de protection avait été construit, qu'un texte seul, aujourd'hui, mentionne, et qu'une grosse campagne de construction, à la fin de la guerre, avait totalement reconstruit. Il n'en reste plus rien, donc, y compris d'un point de vue archéologique, sinon ce document. Si le document avait disparu, qu'en saurions-nous ?

${ }^{7}$ A.N. Fonds Doat, reg. 199, fo 109 à 176 : «Informations faites pour Jean comte d’Armagnac et de Rodes en vertu des letres de commission de Jordain seigneur de Tornamire senechal de la Comté de Rodés sur les dommages, ravages, meurtres et enlevemens faits par les Anglois et ceux de leur parti dans les terres de la dite Comté pendant la treve faite entre les Roys de France et d'Angleterre » (1375).
} 
quandam filium dicti loquentis aprionaverunt, et stacaverunt, post modum ipsum dimiserunt ; plura non deposuit » $\left(\mathrm{f}^{\circ} 170 \mathrm{v}^{\circ}\right.$ et $\left.171 \mathrm{r}^{\circ}\right)$. Ou encore, autre exemple, "Bernardus Salavert dicti mansi de Cossana iuratus ad sancta Dei evangelia dicere veritatem super contentis in dicto titulo, suo iuramento dixit, quod quadam die, de qua dixit se non recordari gentes armorum existentes in dicto loco vocato de Carlat, ad suum bospicium venerunt, et ipsum apraesonaverunt, et liguaverunt, et secum duxerunt, et plura alia mala in suo bospicio dederunt, quod non esset sibi opus, prout dixit pro decem franchis, tamen suo iuramento dixit quod a die sexta iulii fuit factum 》 ( $\mathrm{f}^{\circ}$ $175 \mathrm{r}^{\circ}$ et $\mathrm{v}^{\circ}$ ). Dans les deux cas, une personne au moins est présente dans la maison au moment où les ennemis y pénètrent. Elles n'étaient donc pas dans un lieu refuge, à l'abri de l'exaction. Sans doute l'effet de surprise a-t-il pu jouer: quelles informations étaient-elles disponibles ? qui en disposaient? Une attaque éclair, menée par quelques hommes, pouvait-elle donner lieu à une alerte? Tout un questionnement se fait jour. Il oblige à nous interroger profondément sur notre manière d'appréhender la mise en défense et sur le caractère systématique qu'on lui prête sans le dire : l'habitant d'un écart, au fond de la vallée du ruisseau de Perse, près d'Espalion, avait-il quelque chose à craindre?

Concluons. La mise en défense des communautés est a priori une question connue et relève à l'heure actuelle d'un champ historiographique largement labouré. À bien y regarder de près, pourtant, que sait-on vraiment? La manière dont les villes, petites ou grandes, se protègent. On sait assez bien aussi ce que le château représente et quel rôle il a pu jouer à la fois dans la défense des populations et dans la définition du maillage défensif. Des pans entiers de la question restent dans l'ombre, faute de documentation sans doute, faute d'investigations surtout. En témoigne la mise en place récente de groupes de recherches sur les forts villageois, phénomène encore mal appréhendé : à Toulouse, dans le cadre du regroupement des médiévistes Terrae; en Auvergne, sous l'impulsion de Gabriel Fournier (Fournier, Lorgeoux et Vernet 2000). Une révision complète de ce que l'on sait sur les églises fortifiées s'impose - le dossier est rouvert pour le Rouergue et le Languedoc-Roussillon (Crozier en cours). Plusieurs thèses en cours, pour le Sud-Ouest, s'efforcent d'aborder le problème de la mise en défense dans son ensemble, ou sous un angle neuf (Loppe en cours, Savy en cours, Ferrand en cours). Pour autant, certains aspects de la question continueront d'échapper aux chercheurs, mais qu'il faut bien se garder de perdre de vue. Au final, les yeux rivés sur la documentation disponible - et presque sur elle seule -, les historiens se sont-ils donnés les moyens d'appréhender le phénomène de la mise en défense? Le schéma établi n'est sans doute pas faux; sans doute n'est-il pas à remettre totalement en question. Mais la séparation entre deux mondes, le monde urbain d'un côté et le monde rural de l'autre, deux mondes qui n'en font qu'un, est de mauvais aloi, et empêche de pouvoir disposer d'une vision globale des choses. À défaut, elle oblitère l'appréhension du phénomène de mise en défense. Des points sont fortifiés - c'est une certitude -, villages, villes, tours, églises ou châteaux, où la population du lieu et des environs peut venir se réfugier. Encore faut-il que la menace soit clairement définie et prise en compte à temps : il y a loin entre une troupe de soldats cherchant à s'emparer d'une forteresse, prête à mettre le siège au pied des murs, que l'on entend venir de loin, et les exactions commises en coup de vent par des hommes d'armes surgis d'on ne sait où, qu'une rumeur signale à tel ou tel endroit, sans bien savoir. La norme, c'est cette insécurité larvée, latente, mal définie. Dans l'incertitude, sans doute opère-t-on un resserrement du maillage défensif - l'exemple de Flaujac en est probablement l'illustration -, multipliant la construction de lieux forts; peut-être vit-on aussi, tout autant, sans trop se soucier de ce qui peut survenir : comment se prémunir contre un coup de main que rien n'a prévu et ne peut prévoir ? On connaît, ici et là, des mesures prises pour vivre au quotidien dans cette insécurité irréductible : des sentinelles sont postées sur des hauteurs pendant que des hommes, en contrebas, fauchent ou moissonnent, par exemple.

Ce constant n'est pas satisfaisant. On ne peut se contenter de dire : ici, on sait; là, on en sait pas, ou pas bien. Paradoxalement, travailler à établir une vue d'ensemble, la plus exhaustive 
possible, si elle oblige à dresser ce constant, offre dans le même temps la possibilité de dépasser le problème, et de le régler en partie. Cette vue, pour incomplète qu'elle soit, permet de mettre au jour différentes manières de faire: celle des habitants des agglomérations, celles de certains seigneurs, celles de petites communautés, qui obtiennent l'autorisation de fortifier leur église ou d'ériger un fort. Appréhendées de la sorte, ces différentes manières de faire définissent autant d'espaces défensifs, qui cohabitent et se surimposent. À défaut d'une documentation explicite, c'est là le moyen de comprendre ce qui s'est joué et de relier entre eux les différents éléments du dossier : les hommes, leurs peurs, la communauté à laquelle ils appartiennent et les structures matérielles qu'ils mettent en place pour se protéger.

Bibliographie.

Alexandre de Paris 1994 = Alexandre de PARIS, Le roman d'Alexandre, Paris, Le Livre de Poche - Lettres gothiques, 1994 [fin XII ${ }^{\mathrm{e}}$ siècle];

Affre $\mathbf{1 8 5 8}$ = Henri AFFRE, Lettres à mes neveux sur l'bistoire de l'arrondissement d'Espalion, Villefranche-de-Rouergue, Veuve Cestan, 1858 (2 tomes);

Bériac et Hanusse $\mathbf{1 9 9 2}$ = Françoise BÉRIAC et Claire HANUSSE, «La construction d'une batterie de machines de guerre à Bordeaux en 1324/5", dans Bergerac et le Bergeracois, Actes du $\mathrm{XLII}^{\mathrm{e}}$ Congrès d'études régionales de la F.H.S.O. (Bergerac - mai 1990), Bordeaux, 1992, p. 165 à 203 ;

Blieck 1998 = Gilles BLIECK, "La mise en défense de Lille en 1452-1453 », dans Noël Coulet et Olivier Guyotjeannin (dir.), La ville au Moyen Age, $120^{\circ}$ Congrès nationale des sociétés historiques et scientifiques (Aix-en-Provence - 1995), Paris, Éditions du C.T.H.S., 1998, t. I, p. 195 à 209 ;

Blieck, Contamine, Faucherre et Mesqui 1999 = Gilles BLIECK, Philippe CONTAMINE, Nicolas FAUCHERRE et Jean MESQUI (éd.), Les enceintes urbaines (XIIT $-X V T^{r}$ siècle), Paris, Éditions du C.T.H.S., 1999 ;

Bloch 1952 [1931] = Marc BLOCH, Les caractères originaux de l'bistoire rurale française, Paris, Armand Colin, 1952 (Oslo/Paris, Les Belles Lettres, 1931);

Butaud 2002 = Germain BUTAUD, «Le coût de la guerre et de la défense dans les villes au bas Moyen Âge : l'exemple de la France du Midi et de l'Italie », dans Denis Menjot et Manuel Sánchez Martínez (coord.), La fiscalité des villes au Moyen Âge (Occident méditerranéen). 3 - La redistribution de limpôt, Toulouse, Privat, 2002, p. 235 à 265 ;

Carnus et Cabanettes 1966 = Marcel CARNUS et Émile CABANETTES, Saint-Côme-d'Olt, Rodez, Éditions Subervie, 1966 ;

Chevalier 1981 = Bernard CHEVALIER, «Le paysage urbain à la fin du Moyen Âge: imaginations et réalités ", dans Le paysage urbain au Moyen Age, Actes du XI ${ }^{\mathrm{e}}$ Congrès des historiens médiévistes de l'enseignement supérieur, Lyon, PUL, 1981, p. 7 à 21 ;

Contamine 1978 = Philippe CONTAMINE, «Les fortifications urbaines en France à la fin du Moyen Âge : aspects financiers et économiques ", Revue Historique, 1978, p. 23 à 47 ; 
Contamine 1999 = Philippe CONTAMINE, "Observations sur le siège d'Orléans (14281429) », dans Blieck, Contamine, Faucherre et Mesqui 1999, p. 331 à 343 ;

Contamine 2002a = Philippe CONTAMINE, «L'impact de la guerre de Cent ans en France sur le « plat pays » et sur la vie au village », dans Desplat 2002, p. 15 à 34 ;

Contamine 2002b = Philippe CONTAMINE, «En guise de conclusion : les villes du Languedoc et la chevauchée d'Édouard, prince de Galles (12 octobre - 28 novembre 1355) », dans Le Blévec 2002, p. 195 à 210 ;

Crozier en cours = Jacynth CROZIER, Les églises fortifiées en Languedoc-Roussillon et en Rouergue, thèse en cours sous la double direction de Michel Fixot et de Laurent Schneider, Université d'Aix-Marseille I ;

Desplat 2002 = Christian DESPLAT (éd.), Les Villageois face à la guerre (XIV $-X V I I I^{e}$ siècle), Toulouse, PUM - Flaran XXII, 2002;

Ferrand 2001 = Guilhem FERRAND, Espalion et ses emirons au Moyen Age : étude bistorique et archéologique, mémoire de maitrise sous la direction de Gérard Pradalié, Toulouse, UTM, 2001 ;

Ferrand en cours $=$ Guilhem FERRAND, Communautés et insécurité en Rouergue à la fin du Moyen $\hat{A g e}$, thèse en cours sous la double direction de Mireille Mousnier et de Jean-Loup Abbé, Université de Toulouse ;

Flandin-Bléty 1979 = Pierre FLANDIN-BLÉTY, Essai sur le rôle politique du Tiers État dans les pays de Quercy et de Rouergue (XIII $-X V^{e}$ siècle), thèse de doctorat d'État en droit, Paris, 1979 ;

Fournier 1966 = Gabriel FOURNIER, «La défense des populations rurales pendant la guerre de Cent Ans en Basse-Auvergne ", Actes du $90^{\circ}$ Congrès national des sociétés savantes (Nice 1965). Section d'archéologie, Paris, Bibliothèque Nationale, 1966, p. 157 à 199 ;

Fournier, Lorgeoux et Vernet $\mathbf{2 0 0 0}=$ Gabriel FOURNIER, Françoise LORGEOUX et JeanPaul VERNET, "Forts et autres fortifications villageoises », Revue d'Awvergne, 2000/1-2, p. 151159 ;

Fournioux $1990=$ Bernard FOURNIOUX, «Un dispositif de protection territoriale et de défense des populations rurales en Périgord au XIII ${ }^{\mathrm{e}}$ siècle », Archéologie médiévale, t. XX (1990), p. 335 à 349 ;

Gaier 1995 [1963] = Claude GAIER, «La fonction stratégico-défensive du plat pays au Moyen Âge dans la région de la Meuse moyenne », Le Moyen Âge, 1963, p. 753 à 771 ; repris dans Claude Gaier, Armes et combats dans l'univers médiéval, Bruxelles, De Boeck Université - Bibliothèque du Moyen Âge 5, 1995, p. 267 à 281 ;

Germain 2005 = René GERMAIN, Chartes de franchises et fortifications au duché de Bourbon, Marsat, "La Source d'Or», 2005 ;

Ginisty 1975 = Albert GINISTY, Estaing : aspects du passé, Rodez, Éditions Subervie, 1975 ;

Hayez 1978 = Anne-Marie HAYEZ, "Travaux à l'enceinte d'Avignon sous les pontificats d'Urbain V et de Grégoire XI », dans La guerre et la paix. Frontières et violences au Moyen Age, Actes 
du $101^{\mathrm{e}}$ Congrès national des sociétés savantes (Lille 1976), Paris, Bibliothèque Nationale, 1978, p. 193 à 223 ;

Hébert 1978 = Michel HÉBERT, Tarascon au XIV siècle. Histoire d'une communauté urbaine provençale, Aix-en-Provence, Édisud, 1978 ;

Lardin 2006 = Philippe LARDIN, «Les transformations de la ville de Dieppe pendant la guerre de Cent Ans ", dans Pierre Bouet et François Neveux (dir.), Les villes normandes au Moyen Âge, Caen, PUC, 2006, p. 75 à 108 ;

Lartigaut 1967 = Jean LARTIGAUT, «Les lieux fortifiés dans la partie occidentale du Quercy au $\mathrm{XV}^{\mathrm{e}}$ siècle », Annales du Midi, 1967, p. 5 à 18 ;

Le Blévec 2002 = Daniel LE BLÉVEC (éd.), Défendre la ville dans les pays de la Méditerranée occidentale au Moyen Âge, Montpellier, Université de Montpellier III / Centre historique de recherches et d'études médiévales sur la Méditerranée occidentale - Monspeliensia medievalia 1, 2002 ;

Leguay 2005 = Jean-Pierre LEGUAY, Les catastrophes au Moyen Âge, Paris, Éditions Jean-Paul Gisserot, 2005 ;

Loppe 2004 = Frédéric LOPPE, "L'abbaye de Lagrasse et ses possessions (Aude) : quelques exemples de mise en défense dans la seconde moitié du XIV ${ }^{\mathrm{e}}$ siècle », Archéologie Médiévale, $\mathrm{t}$. XXXIII (2003), p. 139 à 194 ;

Loppe en cours $=$ Frédéric LOPPE, « La terre crue dans la fortification en Lauragais : l'exemple de Castelnaudary (Aude), vers 1355 - vers 1450 », thèse en cours sous la direction de Michel Fixot, Université d'Aix-Marseille I ;

Mesqui 1979 = Jean MESQUI, Provins. La fortification d'une ville au Moyen Agge, Paris, Arts et métiers graphiques - Bibliothèque de la Société française d'archéologie 11, 1979 ;

Michaud-Fréjaville $\mathbf{1 9 8 1}$ = Françoise MICHAUD-FRÉJAVILLE, «La forteresse d'Orléans $\left(\mathrm{XIV}^{\mathrm{e}}-\mathrm{XV}^{\mathrm{e}}\right.$ siècles)", dans Le paysage urbain au Moyen Áge, Actes du XI $\mathrm{X}^{\mathrm{e}}$ Congrès des historiens médiévistes de l'enseignement supérieur, Lyon, PUL, 1981, p. 61 à 78 ;

Poujol et Simonin 1997 = Jean POUJOL et Michel SIMONIN, La forteresse de Peyrelade (Rivièresur-Tarn), DFS, DRAC Midi-Pyrénées, SRA, 1997 ;

Rigaudière 1993 [1984] = Albert RIGAUDIÈRE, «Le financement des fortifications urbaines en France du milieu du XIV ${ }^{\mathrm{e}}$ siècle à la fin du XV siècle ", Revne Historique, 1984, p. 19 à 95 ; repris dans Albert Rigaudière, Gouverner la ville au Moyen Age, Paris, Anthropos-Economica, 1993, p. 417 à 497 ;

Salamagne 1981 = Alain SALAMAGNE, "Les fortifications médiévales de la ville du Quesnoy », Revue du Nord, t. LXIII, 1981/4, p. 997 à 1008 ;

Savy 2005 = Nicolas SAVY, Cahors pendant la guerre de Cent Ans, Cahors, Colorys, 2005 ;

Savy en cours $=$ Nicolas SAVY, La défense des villes et bourgs du Quercy, aspects militaires et socioéconomiques, thèse en cours sous la direction de Jacky Theurot, Université de Besançon ; 
Sireix 1997 = Christophe SIREIX, Le Parmentier (Roder), DFS, DRAC Midi-Pyrénées, SRA, juin 1997 ; 
ANNEXE.

\title{
Instructions et advis pour fermer, clore et armer une ville tels qu'Aristote envoia a Alexandre pour fermer la ville de Bussifali et les autres qu'il aimait :
}

\author{
A. Original perdu. \\ B. Archives Nationales, Fonds Doat, registre 147, $\mathrm{f}^{\circ} 282 \mathrm{r}^{\circ}$ à $287 \mathrm{r}^{\circ}$.
}

Ayssi es la ordonnansa cum una viela se deu fermar et claure et armar contra sos enamixs, laqual trames Aristotial à Alexandre per fermar la sieutat Bussifali et las vielas que amava.

Prumerament deu esser gardat, que i aia pro gens bonas et sufficiens segon la grandor de la vièla. Et aprop, que aio pro vitalha et armaduras et artilharia. Et se aisso, au devo far govern et capitani de un home savi, hardit et leyal, loqual toih crezo. Et aquel deu obrar am lo Cosseilh dels plus savis. Et aquel deu regardar las causas dessus dichas, seys pro gens de bon coratge et vitailha et arma. Et aprop deu a ordenar las gardas de portas et intradas et de yssidas de tours et de cranels. Et far conestablias et centeniers et cinquanteniers et dexeniers.

La maniera fermadura de la viala : /// $\mathbf{f}^{\circ} 282 \mathbf{v}^{\circ} / /$

Prumerament deu aver a tot lo tourn grans valatz lanes et persons tant persons que tengüas esqitas et en las partz on non pot aver aygua deu esser fach en la sola delt valat gran quantitat de vossas cubertas am murailha de terra et d'erba. Et aprop deu aver grans murs et aut am tors bataillueyras de dex en dix brassas, et que lou valat sia bé rasclats, et curats, dal pé del mur tro en la sola d'erbas et de ramailha, et als portals et a las intradas deu aver ponts levadits. Et totz los camis de las intradas devo esser romputz a travers de grands valats en cinq ou en sieys luoxs forque un petit estreiti passatge, et aquel se deu rompre quan besonh es perque a caval ni á pé no se puesquo apropchar de las portas ni fuoc amenar en careixs ni en als. Et far gran re de bosias per los camis de las intradas. Et per dessus los dentilhs dels murs a tot lo torn de la viela deu aver barbaquanas coratanas que passo tres pés fore dels murs cranelat de bois am portanels levadits per on se trazo peiras al pe dels murs, et se no i pot aver tant de barbacanas que aia arequieiras tourneiadisses $/ / \mathbf{f}^{\circ} 283 \mathbf{r}^{\circ} / /$ de bois entre dos cranels. Et de quinze en quinze brassas, tors de bois que s'apelo aguassiers foratanas, que sian auts plus que los cranels per metre las espingnalas els canos et las balestas de torn. Et aprop a cascu dentilh alen deforas deu estar en penden una redondela o una ucha facha de quoatre potz plena de terra, et de peyra et deu esser lhiats als dis caps am una corda, et per dedins lo cranel aquela corda estaquada en un luoc, a la fi que en un cop poguos esser trenquada se nulh venia al pe del mur.

Item per dedins a tot lo torn dels murs sus als cranels, et en las barbacanas deu om metre gran quantitat de peiras ponhals per lansar am fondas que se teno am bastos et ganre d'autras peyras grossas a tot lo torn dels murs per dedins deu aver espazi de quinze pés per passar tres homes a caval lo un costa l'autra. Et deu esser a ordonnat quen cascu cranel estio tres ou quoatre homes armats, que los us aion las ditas fondas per lansar las ditas peiras, ponhals quand besoinh sera. Et trago petits topis plés de cauts viva et d'autras plés de calcatrepas cubertz de tela // $\mathbf{f}^{\circ} 283$ v // et petits crugos plés d'aigua bulhen mesclada dain cautz viva. Et ayssó deu se lansar lay on a gran re de gens de caval ou de pé et los autras compahos dessus los cranels devo aver balestas de torn et d'autras de un pé et canos que ietto guarróts am poudres et espinguales per traire sus los anemixs. Et a cascun cranel, deu aver un mailh de bois am long margue et se d'aquels non a om pro què fasson aportar aquels dels fustiers que fau los cubels. Et los grands martels de fer dels fabres et grandes d'estrals et becuas per que que negus montava sus el mur am escalas ou en altra maneira que de tailhs mailhs fossen feritz per la testa et ia tant no seria armats que no tombes. Et 
devo aver en cascu cranel un petit gaf am que botesso ou tiresso la pes o colpes se i eron aturadas als murs. Et per dedins en cascune part on hom pensa que fa besoinh, deu aver grans engienhs, et peiriers a treire en las vengudas et en las parts on se pot metre seti et soin far anar cascus en sa garda et far en sa garda cascu las bedas los canos, las balestas et tots los autres esturments per que que non ho aio pas a // $\mathbf{f}^{\circ} \mathbf{2 8 4} \mathbf{r}^{\circ} / /$ penre al besonh. Et avisar totz un apres l'autra et tots en poblicq que per critz ni per yschioles ni per brams a mort a mort á l'espaza ni per gran resonamen de trompas ni de grailes ni de claros ni de nacaris nulh no es baica ni fugua de sa garda a pena de la testa ans aio plus fort cor que davan.

Item deu esser a ordenat que el cas on ceria besonh et los enamixs serian pres, que per defors els camis et en las intradas et en la sola dels valats fossen semenada qualque trapas prop de la viela et al besoinh mezeis deu esser a ordenat per dedins que prop dels murs de detz en detz brassas sian mes pairols grans ples d'aigua sus el fuoc et tot iourn boilhir am cautz et sion apareilhatz las cordas els ferratz per gitar sobre aiquels que veinrio al pé dels murs et a cascuna porta per dedins gran re de cubas pleinas d'aigua et las cordas els ferratz estien apareilhatz per lansar sus lo fuoc se los enamixs lo portavo et dessus los dentilhs de trenta en trenta brassas fachas habitaclas de tela enserada per celar can plus $/ / \mathbf{f}^{\circ} \mathbf{2 8 4} \mathbf{v}^{\circ} / /$ la artilharia et per celar la nueich que fan lo gaih sus los murs.

Item deu esser anordanat que quand seria besoinh que los anemixs seriau davan, que per dedins des trenta en trénta brassas estesson cinq ou sieys homes a caval armatz ces mouré de un luoc pé a sortir et cofortar aquels dessus los murs per que no fugo ny sesbais quo. Et se aquels de caval vezian que aquels desus ces baisso per cop ou per nafra ou per forsa de gran assaut que tantost ni trameto d'autras ou que dus outres de lor montesso ou que un ou dus dels autres ano querro aiuda se besonh es.

Item aprop deus anar per viela très ou quoatre companhas d'hommes a caval armatz cascuna de oeyt ou de dex homes et devo anar de la una porta a l'autra et de una garda a d'autra per visitar et afortir et cofortar totas gens et per amenar gens et esfors lai on se donno los assautz et far lor portar armaduras et artilharia et quo comando a pena de la testa, que nulh no se mena de sa garda da nuech ni journ per menjar ni per beure ni per nul besoinh $/ / \mathbf{f}^{\circ} 285 \mathrm{r}^{\circ} / /$ entro que un autra vienha en son luoc esse venso et lasso lor enamixs quoate ou sieys assautz que pertant no se orgoilhosisco ni laisso lor garda ans sa fortisco et se doubte plus que d'avan et en aisso devo aiudar totas las femmes per bonas que siau ben et alegramen et a fortidamen, so es assaber que aporto peyra et aminis tro l'aiga bulhen et freia ou la cautz, et tot so que om lor commandara per riqua que sia et que aporto a tots pas et vis et viandas sus los murs et lay on serau en las gardas per lor cofortar et per que no se parto de lor gardas quar aisso es lor mestier. Et be lor es meilhor que servo los lor essels que las gardo que estar en la merré de lors anemixs. Et per aver meilhor esfors et aiuda deu hom aver tots los hommes dorde dedins la viela et fan armar capelas et clercs, morgue et canorguas, et totz religios et a cascu commandar que fassa. Et si non an armaduras, que la viela lor ne bailla, et que totz obriers ou manediers aio armadura per lor cors et tots autras menestairals. Item las cridas que son // $\mathbf{f}^{\circ} 285 \mathbf{v}^{\circ} / /$ fachas per anan per avan quant hom se doubte de sos anemixs fa hom cridar que tout hom se apareilhe en armas et en cavals et en arneis et a ne armatz segon son poder a peine. Et que nul maisso, peirer ni afanaire non obre mas a la obra de la viela.

Item que totz hom que sabra jogar d'arcq ou de balesta ou d'autras armaduras, que ensenhe los autras. Item que totz juecs de datz et tras gitadors et encantadors de caireforc et beveria de taverna et joglors de samphonia et sturmens et dansas et corolas et tot juec de pilota et totz autres juexs arestost et a presen què tòtz cesson a pena gran. Item que tota faida ou malvolensa que lur aia am l'autra que toih cesso a peina seno lo iuec de laro ou de balesta et que nulh menestairal, fabre, tornor ou fustier, cordurier, balestier ou armurier ou d'autra mestier non obro sino d'artilharia. Et nulh tavernier non et fra juec ni beveria en taverna. Et en special tot juec arestost et a presen sia defendut dus iours avan Nadal et lo iorn et quinse // $\mathbf{f}^{\circ} 286 \mathbf{r}^{\circ} / /$ jorns en apres, a pena. Et als sermonadors en gleisa deu esser comandat que no sermono mas em breus 
paraulas que hom pregues Dieu quens done pats quar en tals iourns a hom plus de perilh que en autre et on plus a om de dopte que adonc deu hom far cridar que nulh estatga de la viela nou prengua viatga ni ane en perelinage ses enprenta del major, a pena, et que nulh mercadier ni obradorier non huebra ni ietx avau nulha deneada a vendre sens armaduras et quar los meilhors jorns au plus de doubte a la dona se deu cridar que cascus sia en armas, a pena. Et a las portas metre gardas.

Item per avan deu hom tener grau re d'espias bonas et certanas et quant hom essertz que elh venò ana que la part, deu lou capitani trametre dos bos savis homès encontra los enamixs sieys ou oeyt legas messatgiers per demandar et saber als capitanis de la ost que volo far ni on volo anar et scaber tot so que poiran de lor volontat et que porto lor rasonier cum aquel luoc no lor á tort et dire so que lo cosseilh // $\mathbf{f}^{\circ} 286 \mathbf{v}^{\circ} / /$ quels tramet lor á comandat et no los detardar tant quant podo a lor demandan acort ou trevus et far lor atendre resposta al plus que podo, et entretan los messatgiers entendo lor volontat et aprendo quan de gens so ni que volo far et devo y retornar motas bets, et entretan las gens se arraon et van en lor gardas et an cosseilh que devo far. Et se no veso lor advantatges, devo penre trevas am argen o ses argen, al plus de temps que podo quar plus vas donar argen qui no $\mathrm{i}$ vesos avantatges que penre lo premier dampnatge, so es assaber dels vilatges et de las borias et dels barris et dels heritatges de foras, part lo gran perilh del tot. Et entretan a hom aut secours á bo cosseilh quar mot deu hom avisar avan la masseya pro gens ny se an bon couratge ni se so be armatz d'armas et de vitailha et que no sian ebronhs ni volpalhs ni davol vida quar gran causa es a fiar a dos ni a tres homes un cranel, als quals no faria aventura trenta libras et en aquest cas sia lor hom tot lo tezaire de una viela et raubas et joyels, crotz, calices, et reilhiquias $/ / \mathbf{f}^{\circ} 287 \mathbf{r}^{\circ} / /$ et perilh del cors encartamens et previlegis de homes et de efans a la honor de las fempnas, per que avan ou deu hom tot compassar quar aprop no val res, mas per res nou deu hom cessar a far totz los emendamens car hom no sap lor a ordonansa et tot jorn es ne hom plus forts et segurs els enamixs plus fibles.

Lo glorious salvador del mon veuilha salvar per sa misericordia la bona viela franca et las bonas gens que y son els bos perregidors que i so ni la governo et totas las autras garde Dieus de perilh selh plahs. Amen omnes. 